\section{Seroprevalence to SARS-CoV-2 Among Healthcare Workers in an Exclusive Pediatric Hospital}

Healthcare workers (HCWs), who are the first line in managing the pandemic of coronavirus disease (COVID-19), have been observed to be at a greater risk of infection [1]. Seroprevalence studies can provide relevant information on the proportion of people who have experienced a recent or past infection. Such studies performed among HCWs can provide information regarding the risk of exposure in a hospital setting, and also about the effectiveness of infection control strategies, including the proper use of personal protective equipment (PPE). Although, several studies have described the seroprevalence among HCWs [2-4], data from an exclusive pediatric hospital is lacking. We attempted to estimate the prevalence of $\mathrm{IgG}$ antibodies against severe acute respiratory syndrome coronavirus 2 (SARS-CoV-2) among $\mathrm{HCW}$, in a private pediatric hospital setting, and to correlate the seroprevalence based on their risk of exposure.

Our hospital is a tertiary care pediatric hospital and is a designated COVID treatment facility since March, 2020. There is regular surveillance of healthcare workers in our institute and quarantine of HCWs with exposure/symptoms were done as per guidelines by the Ministry of Health and Family Welfare [5]. This study was approved by the institutional ethics committee. HCWs who consented to the study were recruited. Sample collection was done between 30 July and 7 August, 2020. We used Indian Council of Medical research (ICMR) approved YHLO SARS- CoV-2 IgG antibody titer assay kit (Shenzhen YHLO Biotech Co. Ltd), and titers above $10 \mathrm{AU} / \mathrm{mL}$ were considered significant. Health care workers were split into two groups, High Risk and Low Risk [2]. HCWs in direct contact with a suspected or confirmed case of COVID-19 were categorized as high risk, and those not in direct contact were categorized under the low-risk group.

Out of the $466 \mathrm{HCWs}$ in our hospital, 95 [41 doctors, 39 nurses, and 15 others (laboratory technicians, nursing orderlies)] participated in the study. Sixteen (16.8\%) tested positive for SARS CoV-2 IgG antibodies. Of the 16 seropositive HCWs, 12 (75\%) reported symptoms compatible with COVID-19 in the past, and 4 (25\%) were asymptomatic. The majority of the seropositive were doctors $(n=8,19.5 \%$ of tested), followed by nurses ( $n=7,17.9 \%$ of tested), with one laboratory person testing positive.

All 12 symptomatic HCWs underwent RT-PCR for SARSCoV-2 and 9 were positive. Among the $95 \mathrm{HCWs}$ participating in the study, $36 \mathrm{HCWs}$ were grouped under high-risk. The proportion of those with positive serology was not significantly different between the two groups $(13.8 \%$ vs $18.6 \% ; P=0.75)$.
In our study, we noted $16.8 \%$ seropositivity to SARS-CoV2 among HCWs. The seroprevalence rates may vary depending on various factors such as awareness and implementation of proper infection control strategies, access to PPE, and community prevalence of the virus. Interestingly we observed that the proportion of seropositivity among HCWs in a highrisk setting was not significantly different than those working in a low-risk setting. The seroprevalence of $16.8 \%$ to SARS CoV 2 among our HCWs is nearly similar to the recently reported community seroprevalence $(21.5 \%)$ in Chennai [6]. We speculate that this might be because HCWs tend to take more precautions in a high-risk exposure setting and the strict implementation of the WHO protocol for PPE [7] in our SARI wards and fever triages. In an earlier observation by Hunter, et al. [8], HCWs with the most exposure to COVID-19 patients were not at higher risk for developing antibodies than HCWs with little to no work-related COVID-19 exposure.

There are a few limitations to our study. We included consenting HCWs in our study rather than by randomization, which could have resulted in those with prior symptoms or exposure volunteering to get tested. We did not assess the source of infection or contact in those who were seropositive. To the best of our knowledge, this is the first study on seroprevalence among HCWs in an exclusive pediatric hospital.

Acknowledgements: Dr Anand Manoharan, Director Clinical research, CTMRF for aiding us in the project and Dr Aishwarya Venkataraman for helping us conduct the tests.

Ethical clearance: CTMRF-KKCTH Ethics Committee.

Contributors - JS, SB- Conceptualization of the study; MM, JS, KD, SP, SB- Writing the manuscript and review of literature; MM, JS- Statistical analysis. All authors approved the final version of the manuscript

Funding: None; Competing interests: None stated.

Published online: January 04, 2021; PII: S097475591600279

MANOJ MAdhUSUdan, ${ }^{1 *}$ JANANi SANKAR ${ }^{1}$, K DhanalaKshmi ${ }^{1}$, Sullochana PUTlibai ${ }^{2}$ and S BALASUBRAMANIAN ${ }^{1}$

${ }^{1}$ Departments of Pediatrics and ${ }^{2}$ Microbiology, Kanchi Kamakoti CHILDS Trust Hospital, Chennai,

Tamil Nadu, India.

*manoj-93@live.in

\section{REFERENCES}

1. Nguyen LH, Drew DA, Graham MS, et al. Risk of COVID-19 among front-line health-care workers and the general community: A prospective cohort study. Lancet Public Health. 2020;5: e475-83.

2. Goenka M, Afzalpurkar S, Goenka U, et al. Seroprevalence of COVID-19 amongst health care workers in a tertiary care hospital of a metropolitan city from India. J Assoc Physicians India. 2020;68:14-19.

3. Korth J, Wilde B, Dolff S, et al. SARS-CoV-2-specific antibody detection in healthcare workers in Germany with direct contact to COVID-19 patients. J Clin Virol. 2020; 128: 104437.

4. Baggett TP, Keyes H, Sporn N, Gaeta JM. Prevalence of SARS- 
CoV-2 infection in residents of a large homeless shelter in Boston. JAMA. 2020; 323: 2191-2192.

5. Advisory for managing Health care workers working in COVID and non-COVID areas of the hospital. Accessed on October 22, 2020. Available from https://www.mohfw.gov.in/pdf/Advisory formanagingHealthcareworkersworkinginCOVIDandNon COVIDareasofthehospital.pdf

6. Fifth of Chennai residents exposed to Covid: Survey. Accessed on November 20,2020 Available from https://www.hindustantimes. com/india-news/fifth-of-chennai-residents-exposed-to-covidsurvey/story-bYJePr6f TEsqrNh8VB3c4I.html
7. Infection prevention and control during health care when novel coronavirus (nCoV) infection is suspected. World Health Organization. Accessed on December 23, 2020. Available from: https:// www.who.int/publications-detail/infection-preventionand-control-during-health-care-when-novel-coronavirus-(ncov)infection-is-suspected-20200125

8. Hunter BR, Dbeibo L, Weaver C, et al. Seroprevalence of severe acute respiratory coronavirus virus 2 (SARS-CoV-2) antibodies among healthcare workers with differing levels of coronavirus disease 2019 (COVID-19) patient exposure. Infect Control Hosp Epid. 2020;41:1441-42.

\section{Digestive Tract Injuries Caused by Ingested Foreign Bodies Containing Magnets}

We report on 16 children with ingestion of magnetic foreign bodies, who were identified by a medical record review of our hospital data for the time period between January, 2017 and May, 2018. Digestive tract wall was sandwiched in $13(75 \%)$ children and 11 (74\%) had gaptic intestinal perforation.

Keywords: Toys, Unintentional injuries.

The ingestion of magnetic foreign bodies in children often requires urgent management, which is quite often surgical [1]. A retrospective analysis was conducted on 16 patients (10 males), who were admitted to our hospital from January, 2017 to May, 2018, and presented with digestive tract injuries caused by magnetic foreign bodies ingestion. The age of these patients ranged within 2-9 years old (median: $4.6 \pm 0.5$ years old), with 6 $(37.5 \%)$ infants and 6(37.5\%) infants.

An abdominal $X$-ray was used to detect the foreign bodies. Appropriate measures were taken for the removal of these foreign bodies. Two patients had removal using foreign body forceps, three patients underwent removal surgery with the use of magnetic attraction, (cylindrical magnets were bound at the end of the snare), and 11 patients underwent laparotomy and repair of the digestive tract.

The foreign bodies were all magnetic components, and $50 \%$ of these foreign bodies were buckyballs. The digestive tract walls of 13 patients $(81.2 \%)$ were sandwiched by the attraction of magnetic foreign bodies, and were injured. Gastric intestinal perforations presented in 11 patients $(68.8 \%)$, while one patient had 20 magnetic foreign bodies located in seven different sites of the small intestine, causing seven perforations in the small intestine.

Abdominal pain $(31.2 \%)$, vomiting $(12.5 \%)$, or both $(37.5 \%)$ were the commonest complaint. In one patient, a tiny "gap" (Fig. 1) in the middle of the foreign body was noted by $X$ ray, and the small intestine walls were sandwiched by the attraction of two small magnetic foreign bodies, resulting in the perforation of the small intestine. All patients had a satisfactory outcome. Five patients underwent gastroscopic removal of foreign bodies and 11 patients underwent laparotomy for removal surgery.
With magnetic foreign bodies, gastrointestinal pressure necrosis between foreign bodies and the formation of fistula can occur [2]. The diagnosis of magnetic foreign bodies in the digestive tract is mainly dependent on the medical history, and the abdominal $X$-ray. Both anteroposterior and lateral $X$-ray films of the abdomen must be combined with careful examination, in order to determine whether a tiny 'gap' in its middle is present for the single metal foreign body in a fixed position of the digestive tract. Therefore, for multiple small adhesive metal foreign bodies revealed by an abdominal $X$-ray film, it should first be considered whether these are connected by the attraction among these magnetic foreign bodies, and whether the digestive tract walls are sandwiched by the attraction of these foreign bodies.

Upon magnetic approximation (when more than one foreign body is ingested), a considerable amount of force can result in inseparable magnetic attraction between bowel loops [3], which would rapidly result in the necrosis and perforation of the intestines.

Conservative treatment may be appropriate for patients who have ingested a single foreign body [4,5]. Although foreign body forceps under a gastroscope cannot effectively grasp these foreign bodies, based on the experience of the investigators, this can be performed as long as the connection point between the two magnetic foreign bodies formed by the magnetic attraction is grasped by the foreign body forceps.

Magnetic foreign body ingestion has typical features on the abdominal $X$-ray and early laproscopic/surgical intervention leads to a good outcome.

Ethics approval: Ethics Committee of The General Hospital of Ningxia Medical University; No. 2019-388.

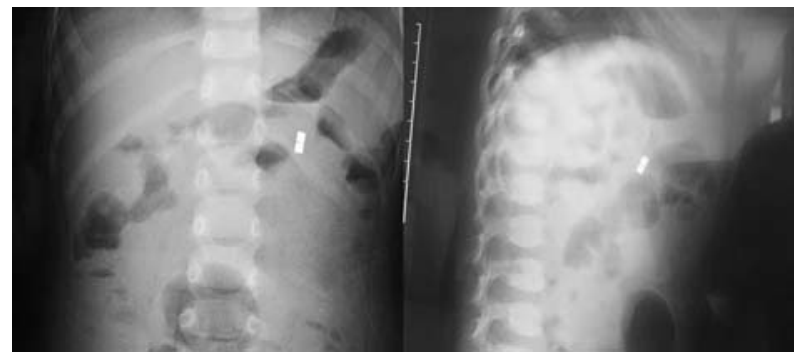

Fig. 1 The anteroposterior and lateral $X$-ray films of the abdomen, showing a 'gap' among the small magnetic foreign bodies. 